\title{
Three-dimensional Yang-Mills-Chern-Simons theory from a D3-brane background with D-instantons
}

\author{
Si-wen Li®, ${ }^{*}$ Sen-kai Luo, ${ }^{\dagger}$ and Mu-zhi Tan ${ }^{*}$ \\ Department of Physics, School of Science, Dalian Maritime University, Dalian 116026, China
}

(Received 11 June 2021; accepted 10 August 2021; published 7 September 2021)

\begin{abstract}
By constructing the configuration of D3-branes with D(-1)-branes as D-instantons, we study the threedimensional Yang-Mills-Chern-Simons theory in holography. Because of the presence of the D-instantons, the D7-branes with discrepant embedding functions are able to be introduced in order to include the fundamental fermions (as flavors) and the Chern-Simons term (at very low energy) in the dual theory. The vacuum structure at zero temperature is studied in the soliton background, and it illustrates the topological phase transition in the presence of instantons. Moreover, since the confinement/deconfinement phase transition could be holographically identified as the Hawking-Page transition in the bulk, we accordingly calculate the critical temperature of the deconfinement phase transition by collecting the bulk on-shell action as the thermodynamical free energy. On the other hand, we evaluate the difference of the entanglement entropy in slab configuration by using the Ryu-Takayanagi formula since the confinement may also be characterized by the entanglement entropy. Altogether we find the behavior of the critical temperature is in qualitative agreement with the behavior of the critical length determined by the entanglement entropy which implies the entanglement entropy could indeed be a character of the confinement in our setup and the D3-D(-1) system would be a remarkable approach to study the threedimensional gauge theory.
\end{abstract}

DOI: 10.1103/PhysRevD.104.066008

\section{INTRODUCTION}

In the past decade, a specific class of three-dimensional (3D) Chern-Simons (CS) theory involving fundamental matters with $N_{f}$ flavors attracts many interests and the large $N$ 't Hooft limit of such theories with a $U(N)$ gauge symmetry has been studied in detail [1-7]. It is conjectured there would be a conformal field theory living in the limit of vanishing flavor mass. Along this direction, some evidence was found that may be supportable to the conjecture, e.g., boson/fermion duality [8] and level/rank duality [9]. On the other hand, the large $N$ field theory can be analyzed holographically by using string theory according to gauge/ gravity duality or AdS/CFT correspondence, which has become very famous nowadays $[10,11]$. Therefore investigation of the CS theory in holography naturally becomes a remarkable topic. And in this work, our goal is to explore

\footnotetext{
*siwenli@dlmu.edu.cn

†1uosenkai@dlmu.edu.cn

tanmuzhi@dlmu.edu.cn
}

Published by the American Physical Society under the terms of the Creative Commons Attribution 4.0 International license. Further distribution of this work must maintain attribution to the author(s) and the published article's title, journal citation, and DOI. Funded by SCOAP. an exactly holographic description of 3D theory with a CS term.

In string theory, the simplest way to build a holographic duality for 3D nonsupersymmetric theory is to construct the configuration of coincident $N_{c}$ D3-branes, i.e., to compactify one of the spatial directions of the D3-brane on a supersymmetry breaking circle $S^{1}[12,13]$. Such a configuration of $N_{c}$ D3-branes at the large $N_{c}$ limit is described by IIB supergravity, and it has been attempted to study the 3D YangMills-Chern-Simons (YMCS) theory or 3D QCD $\left(\mathrm{QCD}_{3}\right)$ in holography, e.g., the vacuum structure $[14,15]$ and the quantum Hall effect [16]. However, the D3-brane background does not include dynamical D-instanton, which would play the important role to involve the topological properties in the dual theory. For example, as it is known the YM instanton in 4D quantum chromodynamics (QCD) is topologically nontrivial excitation of the vacuum that contributes to the thermodynamics of the theory and relates to chiral symmetry breaking $[17,18]$. Based on string theory, the $\mathrm{D} p-\mathrm{D}(p-4)$ brane system [ $N \mathrm{D} p$-branes and $M \mathrm{D}(p-4)$ branes] has gauge theory instantons with exactly $4 N M$ moduli as in gauge theory $[19,20]$; thus, via gauge/gravity duality, a holographic way to include the YM instanton is to take into account the configuration of coincident $N_{c}$ D3-branes (choosing $p=3$ ) with $N_{\mathrm{D}} \mathrm{D}(-1)$-branes as D-instantons smeared homogeneously in the world volume of the D3-branes [21-23]. The system of D3-branes with 
dynamical D-instantons succeeds to describe the 4D QCDlike theories, and the presence of D-instantons could reflect some topological properties of the dual theory [24-27]. So the D3-D(-1) approach has provided an impressive interpretation of the D-instanton.

In order to work out a holographic description of 3D theory with a CS term, in this note we focus on constructing the D3-D-instanton configuration by following the standard technique $[12,13]$ in string theory because the D3-D(-1) approach would turn out that the $\mathrm{D}(-1)$-branes, as the D-instantons, could reduce to a CS term in the 3D dual theory. Specifically, since the $\mathrm{D}(-1)$-branes are dynamical in the supergravity background, there would be a nonvanished Ramond-Ramond zero form $C_{0}$ coupling to these $\mathrm{D}(-1)$-branes. So when we examine the dual theory by considering a probe D3-brane, its action would contain a term as $\int C_{0} F \wedge F$. As we will focus on the 3D dual theory obtained by compactifying one of the spatial directions on the circle $S^{1}$, thus below the energy scale of the circle, the term $\int C_{0} F \wedge F$ can be integrated out to become a 3D CS term as $\int C_{0} F \wedge F \sim \int d C_{0} \wedge \omega_{3} \sim \frac{k_{b}}{4 \pi} \operatorname{Tr} \int \omega_{3}$ where $\omega_{3}$ refers to the CS three-form and $k_{b}$ corresponds to the boundary value of $C_{0}$. Afterwards once the soliton anti-de Sitter (AdS) solution is chosen, it will lead to an area law due to the asymptotics of the Wilson loop, so confinement is also expected to exhibit in the dual theory at low temperature. To further include matters, the flavors are introduced by embedding a stack of probe D7-branes [28] and the spontaneous breaking of chiral symmetry can be identified as the separation of $N_{f}$ flavor branes in the IR region depicted by the IIB supergravity as the holographic description of [29]. Moreover, additional D7-branes as CS branes with a discrepancy embedding function can be put into the background due to the presence of the D-instantons; accordingly at very low energy, the dual theory could be a pure CS theory. We specifically analyze the behaviors of the embedding functions of the various D7-branes and evaluate the associated free energy density by including both flavors and CS terms. The result illustrates the topological phase transition that is enhanced by the D-instantons, and it seems to be qualitatively consistent with what the presence of a CS term topologically contributes to the mass parameter [30] and the D0-D4 approach in 4D [31-34].

Besides, we evaluate the critical temperature of the deconfinement phase transition in this holographic setup since the dual theory is expected to exhibit the confinement. While the deconfining geometry in holography is less clear, the phase transition at a critical temperature can be identified as the bubble/black brane transition, or namely Hawking-Page transition, in the bulk which is very suggestive of the deconfinement transition [35-38]. Following this idea, we collect the total on-shell action as the holographic free energy consisted of the bulk fields (as the color sector), the flavor and the CS branes, in the soliton and black brane background respectively. By comparing the free energy, we find the charge density of D-instantons indeed contributes to the thermodynamics and the critical temperature is decreased by the D-instantons through the flavor and CS branes. However, at the large- $N_{c}$ limit, the critical temperature becomes independent on the D-instantons since the backreaction of the CS branes is not included in this setup. To parallel examine whether our analysis for the deconfinement transition is consistent, we on the other side calculate the entanglement entropy holographically in this system since the entanglement entropy may also be a characteristic tool to detect the confinement in the dual theory [39-42]. Using the Ryu-Takayanagi (RT) formula [43] with the slab geometry, the holographic entanglement entropy can be obtained, and it exhibits a first order phase transition at a critical length which behaves similarly as the critical temperature evaluated by the thermodynamics. In this sense, we believe the configuration of D3-branes with D-instantons would be a remarkable approach to study 3D gauge theory.

The outline of this note is as follows. In Sec. II, we construct the black D3-D(-1) solution to obtain a confining geometry and examine the dual theory by a probe D3-brane. Afterwards, we analyze the embedding function of the flavor and CS branes, compute the free energy by including the flavor and CS term in Sec. III, and then evaluate the vacuum structure of the dual theory in Sec. IV. In Sec. V, we investigate the deconfinement phase transition by comparing the free energy of this model thermodynamically and compute the variation of the entanglement entropy as a parallel verification. Summary and comments are given in the last section.

\section{THREE-DIMENSIONAL THEORY FROM CONFINING GEOMETRY}

In this section, we will briefly review the system of $N_{c}$ D3-branes with $N_{\mathrm{D}}$ D-instantons, i.e., the D(-1)-branes, and then construct the background geometry for a confining dual theory at the large- $N_{c}$ limit.

The D3-D(-1) brane system is geometrically represented by a deformed D3-brane solution with a nontrivial Ramond-Ramond (R-R) scalar field $C_{0}$ which is recognized as a marginal "bound state" of D3-branes with $N_{\mathrm{D}}$ smeared $\mathrm{D}$ (-1)-branes. We denote the $N_{c}$ D3-branes as color branes. In the large $N_{c}$ limit, the ten-dimensional (10D) type IIB supergravity action, as the effective action, describes the low-energy dynamics of this system which in string frame is given as

$$
S_{\mathrm{IIB}}=\frac{1}{2 \kappa_{10}^{2}} \int d^{10} x \sqrt{-g}\left[e^{-2 \Phi}(\mathcal{R}+4 \partial \Phi \cdot \partial \Phi)-\frac{1}{2}\left|F_{1}\right|^{2}-\frac{1}{2}\left|F_{5}\right|^{2}\right] .
$$


Here $2 \kappa_{10}^{2}=(2 \pi)^{7} l_{s}^{8}$ is the 10D gravity coupling constant, $l_{s}$ and $g_{s}$ are, respectively, the length and the coupling constant of string, and $F_{1,5}$ is the field strength of the R-R zero and four-form $C_{0,4}$. The near-horizon solution of nonextremal D3-branes with a nontrivial $C_{0}$ in string frame reads

$$
\begin{aligned}
d s^{2} & =e^{\frac{\phi}{2}}\left\{\frac{r^{2}}{R^{2}}\left[-f_{T}(r) d t^{2}+d \mathbf{x} \cdot d \mathbf{x}\right]+\frac{1}{f(r)} \frac{R^{2}}{r^{2}} d r^{2}+R^{2} d \Omega_{5}^{2}\right\}, \\
e^{\phi} & =1+\frac{Q}{r_{H}^{4}} \ln \frac{1}{f(r)}, \quad f_{T}(r)=1-\frac{r_{H}^{4}}{r^{4}}, \quad F_{5}=d C_{4}=g_{s}^{-1} \mathcal{Q}_{3} \epsilon_{5}, \\
F_{1} & =d C_{0}, \quad C_{0}=-i e^{-\phi}+i \chi, \quad \phi=\Phi-\Phi_{0}, \quad e^{\Phi_{0}}=g_{s},
\end{aligned}
$$

where $\epsilon_{5}$ is the volume element of a unit $S^{5}$ and

$$
R^{4}=4 \pi g_{s} N_{c} l_{s}^{4}, \quad \mathcal{Q}_{3}=4 R^{4}, \quad Q=\frac{N_{\mathrm{D}}}{N_{c}} \frac{(2 \pi)^{4} \alpha^{2}}{V_{4}} \mathcal{Q}_{3}
$$

This solution describes that the D-instanton charge $N_{\mathrm{D}}$ is smeared over the world volume $V_{4}$ of the coincident black $N_{c}$ D3-branes homogeneously with a horizon at $r=r_{H}$. And it implies $N_{\mathrm{D}} / N_{c}$ must be fixed since the backreaction of the D-instantons has been involved in the bulk action. The dual theory of this system is conjectured as the 4D $\mathcal{N}=4$ super Yang-Mills theory (SYM) in a self-dual gauge field background or with a dynamical axion at finite temperature characterized by the order parameter $Q$. In order to obtain a confining or QCD-like dual theory, let us follow the discussion in $[12,13]$. Specifically we first take one of the three spatial dimensions $x^{i}$ of the D3-branes to be compactified on a circle $S^{1}$ with a period $x^{i} \sim x^{i}+\delta x^{i}$. Hence below the Kaluza-Klein energy scale defined as $M_{\mathrm{KK}}=2 \pi / \delta x^{i}$, the dual theory becomes effectively three dimensional. Then we are going to get rid of all massless particles other than the gauge fields. The simplest way to achieve this is to impose the antiperiodic and periodic boundary condition on fermion and bosonic fields, respectively, along $S^{1}$. So the supersymmetric fermions and scalars in the dual theory acquire mass of order $M_{\mathrm{KK}}$ which is accordingly decoupled in the low-energy dynamics. Next we perform a double Wick rotation on the $\mathrm{D}(-1)-\mathrm{D} 3$ brane background, i.e., $t \rightarrow-i x^{i}, x^{i} \rightarrow-i t$ to identify the bulk gravity solution as its holographic correspondence. Without loss of generality, let us denote the direction along $S^{1}$ as $x^{i}=x^{3}$ throughout this manuscript; thus, the confining solution of nonextremal D3-branes with smeared D-instantons is obtained as

$$
\begin{aligned}
& d s^{2}=e^{\phi / 2}\left\{\frac{r^{2}}{R^{2}}\left[\eta_{a b} d x^{a} d x^{b}+f(r)\left(d x^{3}\right)^{2}\right]+\frac{1}{f(r)} \frac{R^{2}}{r^{2}} d r^{2}+R^{2} d \Omega_{5}^{2}\right\}, \\
& f(r)=1-\frac{r_{\mathrm{KK}}^{4}}{r^{4}}, \quad a, b=0,1,2,
\end{aligned}
$$

where the solution of dilaton $\Phi$ and R-R fields $C_{0,4}$ remains. Since the warp factor $e^{\phi / 2} \frac{r^{2}}{R^{2}}$ never goes to zero, the solution (2.4) defined for $r>r_{\mathrm{KK}}$ does not have a horizon. And it would lead to an area law in the dual theory according to the asymptotics of the Wilson loop in this geometry. Namely below the energy scale $M_{\mathrm{KK}}$, the dual field theory should exhibit confinement. To avoid the conical singularities in the region of $r>r_{\mathrm{KK}}$, we have to further require

$$
M_{\mathrm{KK}}=\frac{2 r_{\mathrm{KK}}}{R^{2}} .
$$

Afterwards, the dual theory can be examined by taking into account the action of a probe D3-brane, which is expected to be a 3D YM plus CS theory at $r \rightarrow \infty$ as

$$
\begin{aligned}
S_{\mathrm{D} 3} & =-\mu_{3} \int d^{4} x e^{-\phi} \operatorname{Str} \sqrt{-\operatorname{det}(g+\mathcal{F})}+\mu_{3} \int C_{4}+\frac{1}{2} \mu_{3} \operatorname{Tr} \int C_{0} \mathcal{F} \wedge \mathcal{F} \\
& \simeq-\frac{1}{2 g_{\mathrm{YM}}^{2}} \operatorname{Tr} \int d^{4} x F_{\mu \nu} F^{\mu \nu}-\frac{1}{4 \pi} \operatorname{Tr} \int d C_{0} \wedge \omega_{3}+\mathcal{O}\left(F^{4}\right) \\
& =-\frac{1}{2 g_{3 d, Y M}^{2}} \operatorname{Tr} \int d^{3} x F_{a b} F^{a b}+i \frac{k_{b}}{4 \pi} \operatorname{Tr} \int_{\mathbb{R}^{1,2}} \omega_{3},
\end{aligned}
$$


where $\mathcal{F}=2 \pi \alpha^{\prime} F$ is the gauge strength, $\mu_{p}=(2 \pi)^{-p} l_{s}^{-p-1}$ refers to the D-brane charge, and $\omega_{3}$ is the Chern-Simons threeform,

$$
\omega_{3}=A \wedge d A+\frac{2}{3} A \wedge A \wedge A .
$$

By imposing the background solution, it leads to

$$
\left.d C_{0}\right|_{r \rightarrow \infty}=-i k_{b} M_{\mathrm{KK}} \delta\left(x^{3}-\bar{x}^{3}\right) d x^{3}, \quad g_{3 \mathrm{D}, \mathrm{YM}}^{2}=\frac{g_{\mathrm{YM}}^{2} M_{\mathrm{KK}}}{2 \pi},
$$

where we have assumed that $\omega_{3}$ is independent of $x^{3}$ and does not have components along $x^{3}$. So (2.6) represents the YM-CS action located at $x^{3}=\bar{x}^{3}$, which means $\left.C_{0}\right|_{r \rightarrow \infty}=$ 0 if $x^{3} \in\left(0, \bar{x}^{3}\right) ;\left.C_{0}\right|_{r \rightarrow \infty} \neq 0$ if $x^{3} \in\left(\bar{x}^{3}, 2 \pi M_{\mathrm{KK}}^{-1}\right)$. In this case, we have to slightly modify the supergravity solution for $C_{0}$ in (2.2) as

$$
\chi=1-k_{b} M_{\mathrm{KK}} \Theta\left(x^{3}-\bar{x}^{3}\right),
$$

where $\Theta\left(x^{3}-\bar{x}^{3}\right)$ is the step function.

\section{FLAVOR AND CHERN-SIMONS BRANE}

In this section, let us discuss the embedding of flavor and CS brane in the D3-brane background with D-instantons (2.4) in holography.

\section{A. Adding flavors}

According to the dictionary of AdS/CFT, introducing flavors into the holographic background is to add fundamental matter in the dual theory [28]. So follow the discussion of the D3/D7 approach, we put a stack of $N_{f}$
D7-branes as probes, as $N_{f}$ copies of fundamental flavors, into our background (2.4), and the configuration of various D-branes is illustrated in Table I. Note that in this configuration the supersymmetry is completely broken even in an extremal D3-brane background since the leftover direction $x^{9}$ is transverse to both flavor D7- and color D3-branes which leads to six mixed Neumann-Dirichlet boundary conditions. The bare mass of flavors corresponds to the separation between color and flavor branes at the UV boundary, which means the scalar field respected to $x^{9}$ on the world volume of the D7-branes is the mass operator $\bar{\psi} \psi$ in the dual field theory.

Since the directions $x^{4} \cdots x^{9}$ transverse to the $N_{c}$, D3-branes are usually described by spherical coordinates; for convenience we introduce a new radius coordinate $\rho$ as

$$
r(\rho)=\left(\rho^{2}+\frac{r_{\mathrm{KK}}^{4}}{4 \rho^{2}}\right)^{1 / 2}, \quad \rho>\frac{r_{\mathrm{KK}}}{\sqrt{2}}
$$

and thus the metric (2.4) on coordinate $\rho$ can be written as

$$
d s^{2}=e^{\phi / 2}\left\{\frac{r^{2}}{R^{2}}\left[\eta_{\alpha \beta} d x^{\alpha} d x^{\beta}+f(r)\left(d x^{3}\right)^{2}\right]+\frac{R^{2}}{\rho^{2}}\left(d \rho^{2}+\rho^{2} d \Omega_{5}^{2}\right)\right\} .
$$

Then let us choose the spherical coordinates $\lambda, \Omega_{4}$ to reparametrize the directions $x^{4} \cdots x^{8}$ which are part of the world volume of flavor branes and separate transverse coordinate $x^{9} \equiv u$ with $\rho^{2}=\lambda^{2}+u^{2}$. Afterwards the metric on $\left\{x^{a}, x^{3}, \lambda, \Omega_{4}, u\right\}$ takes the form as

\begin{tabular}{|c|c|c|c|c|c|c|c|c|c|c|c|}
\hline & -1 & 0 & 1 & 2 & (3) & $4(r)$ & 5 & 6 & 7 & 8 & 9 \\
\hline $\mathrm{D}(-1)$-branes & - & & & & & & & & & & \\
\hline Color D3-branes & & - & - & - & - & & & & & & \\
\hline Flavor D7-branes & & - & - & - & & - & - & - & - & - & \\
\hline CS D7-branes & & - & - & - & & & - & - & - & - & - \\
\hline
\end{tabular}

$$
d s^{2}=e^{\phi / 2}\left\{\frac{r^{2}}{R^{2}}\left[\eta_{a b} d x^{a} d x^{b}+f(r)\left(d x^{3}\right)^{2}\right]+\frac{R^{2}}{\rho^{2}}\left(d \lambda^{2}+\lambda^{2} d \Omega_{4}^{2}+d u^{2}\right)\right\}
$$

TABLE I. The configuration of various D-branes. "-" represents the D-branes that extend along this direction. Note that " -1 " is vertical to all the directions of bulk spacetime. 
where $r=r(\rho)$. Embedding the flavor brane into $\left\{x^{a}, \lambda, \Omega_{4}\right\}$ at a constant $x^{3}$ with $u=u(\lambda)$, the induced metric on the flavor D7-brane becomes

$$
d s_{\mathrm{D} 7}^{2}=e^{\phi / 2}\left\{\frac{r^{2}}{R^{2}} \eta_{\alpha \beta} d x^{\alpha} d x^{\beta}+\frac{R^{2}}{\rho^{2}}\left[\left(1+u^{\prime 2}\right) d \lambda^{2}+\lambda^{2} d \Omega_{4}^{2}\right]\right\} .
$$

Note that we need to impose the following boundary condition $\left(\frac{d u}{d \lambda} \equiv u^{\prime}\right)$,

$$
\left.u^{\prime}\right|_{\lambda=0}=0,\left.\quad u\right|_{\lambda=\lambda_{\infty}}=u_{\infty} .
$$

We have used $\lambda_{\infty}$ to denote the UV boundary or UV cutoff in the dual field theory. So for a single D7-brane, its action can be collected as

$$
S_{\mathrm{D} 7}=-T_{\mathrm{D} 7} \int d^{8} x e^{-\phi} \sqrt{-g_{\mathrm{D} 7}}
$$

where $T_{\mathrm{D} 7}=g_{s}^{-1} \mu_{p}$ is the tension of the $\mathrm{D} p$-brane. Plugging the solution (2.2) into (3.6), the action of a probe flavor brane is obtained as

$$
S_{\mathrm{D} 7}=-T_{\mathrm{D} 7} V_{3} V_{S^{4}} R^{2} \int d \lambda e^{\phi(\rho)}\left(\rho^{2}+\frac{r_{\mathrm{KK}}^{4}}{4 \rho^{2}}\right)^{3 / 2} \frac{\lambda^{4}}{\rho^{5}} \sqrt{1+u^{\prime 2}},
$$

where $V_{3}$ and $V_{S^{4}}$ refer to the Minkowskian world volume of D3-brane and the volume of a unit $S_{4}$. By varying the D7-brane action respected to $u(\lambda)$, the associated equation of motion is

$$
\begin{aligned}
& \frac{d}{d \lambda}\left[e^{\phi}\left(r_{\mathrm{KK}}^{4}+4 \rho^{4}\right)^{3 / 2} \frac{\lambda^{4}}{8 \rho^{8}} \frac{u^{\prime}}{\sqrt{1+u^{\prime 2}}}\right] \\
& =-e^{\phi}\left[r_{\mathrm{KK}}^{4}+\rho^{4}-\frac{1}{8} \rho \frac{d \Phi}{d \rho}\left(r_{\mathrm{KK}}^{4}+4 \rho^{4}\right)\right]\left(r_{\mathrm{KK}}^{4}+4 \rho^{4}\right)^{1 / 2} \frac{\lambda^{4} u}{\rho^{10}} \sqrt{1+u^{\prime 2}} .
\end{aligned}
$$

In order to obtain the embedding function $u(\lambda)$, we have to solve (3.8) with (3.5). So let us analyze massless and massive embedding of the flavor brane, respectively.

\section{Massless case}

First let us investigate the case of the limit $r_{\mathrm{KK}} \rightarrow 0,{ }^{1}$ which corresponds to the extremal D3-D(-1) solution. The equation of motion (3.8) comes to

$\frac{d}{d \lambda}\left[e^{\phi} \frac{\lambda^{4}}{\rho^{2}} \frac{u^{\prime}}{\sqrt{1+u^{\prime 2}}}\right]=-\frac{2 \lambda^{4} u}{\rho^{4}} e^{\phi}\left(1-\frac{1}{2} \rho \frac{d \phi}{d \rho}\right) \sqrt{1+u^{\prime 2}}$,

where

\footnotetext{
${ }^{1}$ Since the value (of, e.g., dilaton) at $r_{\mathrm{KK}}=0$ may be divided, we will not take the strict limit although the limit of $r_{\mathrm{KK}} \rightarrow 0$ is well defined in the D-brane background [12]. An effective way is to choose $r_{\mathrm{KK}}=\varepsilon$ where $\varepsilon$ is infinitesimally small, and then take $\varepsilon \rightarrow 0$ in the final result, so there would be no inconsistency in our calculation.
}

$$
\phi \rightarrow 1+\frac{Q}{r^{4}}
$$

in the limit of $r_{\mathrm{KK}} \rightarrow 0$. It is clear that at $\lambda=0$ the righthand side of (3.9) is not vanished unless $u(\lambda)=0$ is the solution. We expect $u(\lambda)=0$ to be an unstable solution as it is discussed in the D3/D7 approach [14,15] since this solution is invariant under the parity transformation $u(\lambda) \rightarrow-u(\lambda)$.

Then let us investigate the case of $r_{\mathrm{KK}}>0$. In the massless, we need to choose $u_{\infty}=0$ in (3.5) since there is a parity transformation $u(\lambda) \rightarrow-u(\lambda)$ in the massless case and $u_{\infty}$ corresponds to the bare mass of the flavors. In order to search for an analytical solution, we use the following ansatz for $u(\lambda)$ as

$$
u(\lambda)= \begin{cases} \pm \sqrt{\frac{r_{\mathrm{KK}}^{2}}{2} k-\lambda^{2}}, & 0 \leq \lambda \leq \sqrt{\frac{k}{2}} r_{\mathrm{KK}}, \\ 0, & \lambda>\sqrt{\frac{k}{2}} r_{\mathrm{KK}}\end{cases}
$$

where $k \equiv k(q)$ is a constant dependent on $q=Q / r_{\mathrm{KK}}^{4}$ only. Notice that $r \in\left(r_{\mathrm{KK}}, \infty\right)$ so that $k \geq 1$. Plugging 


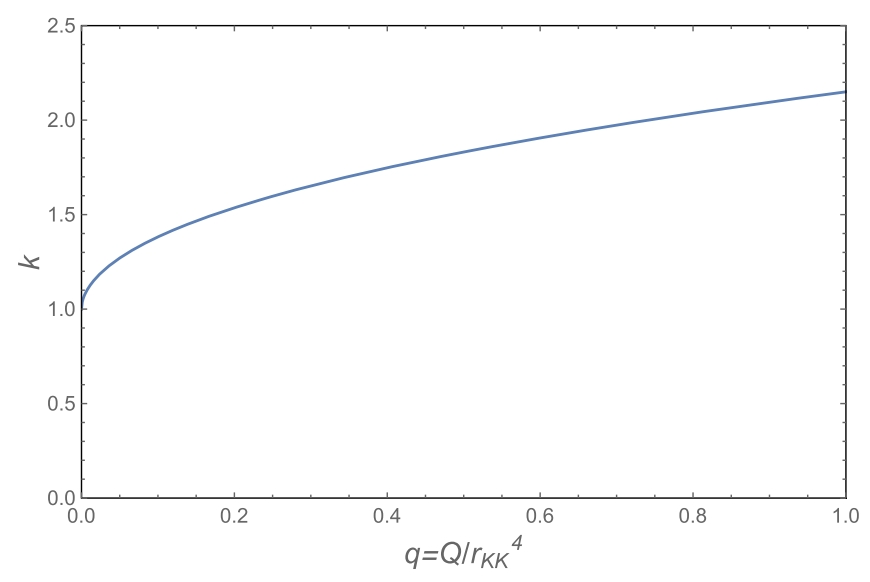

FIG. 1. Numerical solution of $k$ and $q$.

(3.11) into (3.8), it leads to a constraint equation which determines the relation of $k$ and $q$ as

$3+3 k^{4}-2 k^{2}(3+8 q)-3 q\left(k^{2}-1\right)^{2} \ln \left[\left(\frac{k^{2}-1}{k^{2}+1}\right)^{2}\right]=0$.

This equation can be numerically solved, and the relation of $k$ and $q$ is illustrated as in Fig. 1. The solution (3.11) has two branches which refer to a pair of D7-branes wrapping the upper and lower half-five-sphere with various numbers of D-instantons represented by $q$. The flavor branes wrapping the upper and lower half-five-sphere have opposite parity as it is illustrated in Fig. 2. Since the relation of $k$ and $q$ smoothly returns to the case without $\mathrm{D}$-instantons, i.e., $k=1, q=0$, the solution (3.11) corresponds to the maximal embedding of the flavor branes as it is in the D3/ D7 approach, which refers to the embedded flavor branes that have maximal energy among all possible solutions to (3.8) with $u_{\infty}=0$ in the boundary condition (3.5).

In order to find a more general configuration of $u(\lambda)$, let us take a look at the asymptotic behaviors of (3.8). In the

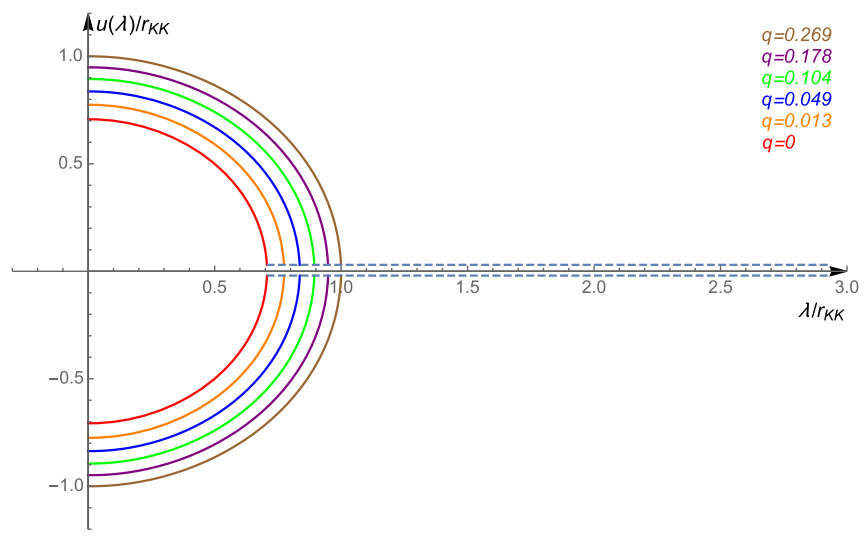

FIG. 2. The maximal embedding of flavor D7-branes in the $u, \lambda$ plane with various $q$. region of $\lambda \rightarrow 0$, we have $u^{\prime}(\lambda) \rightarrow 0, \rho \rightarrow u$, so (3.8) leads to a solution as

$u(\lambda)= \pm u_{0} \mp\left[\frac{4\left(r_{\mathrm{KK}}^{4}+u_{0}^{4}\right)}{5 u_{0}\left(r_{\mathrm{KK}}^{4}+4 u_{0}^{4}\right)}-\frac{1}{10} \phi^{\prime}\left(u_{0}\right)\right] \lambda^{2}+\mathcal{O}\left(\lambda^{4}\right)$.

Note that $u_{0}>0$ and

$$
\begin{aligned}
\phi^{\prime}\left(u_{0}\right) \equiv & \left.\frac{\partial}{\partial \rho} \phi(\rho)\right|_{\rho=u_{0}} \\
= & \frac{64 Q r_{\mathrm{KK}}^{4} u_{0}^{3}}{\left(r_{\mathrm{KK}}^{4}-4 u_{0}^{4}\right)\left(r_{\mathrm{KK}}^{4}+4 u_{0}^{4}\right)\left[r_{\mathrm{KK}}^{4}+Q \ln \frac{\left(r_{\mathrm{KK}}^{4}+4 u_{0}^{4}\right)^{2}}{\left(r_{\mathrm{KK}}^{4}-4 u_{0}^{4}\right)^{2}}\right]} \\
& <0,
\end{aligned}
$$

due to $u_{0}>\frac{\sqrt{2}}{2} r_{\mathrm{KK}}$. The second derivative of $u(\lambda)$ takes the opposite sign to $\left.u(\lambda)\right|_{\lambda=0}$. On the other hand, in the region of $\lambda \rightarrow \infty$, we have $u(\lambda) \rightarrow u_{\infty}, \rho \rightarrow \lambda$, so Eq. (3.8) becomes

$$
\frac{d}{d \lambda}\left(\lambda^{2} u^{\prime}\right)=-2 u
$$

Accordingly, the asymptotic behavior of $u(\lambda)$ at large $\lambda$ takes the general form as

$$
u(\lambda)= \pm \sqrt{\frac{\mu^{3}}{\lambda}} \sin \left(\frac{\sqrt{7}}{2} \ln \frac{\lambda}{\lambda_{\infty}}\right)
$$

by imposing the boundary condition $u\left(\lambda_{\infty}\right)=0$ where $\mu, \lambda_{\infty}>0$ are the integration constants. This solution also has two branches, and thus it implies the global signs of $u(\lambda)$ and $u^{\prime}(\lambda)$ are opposite as well. Keeping this in mind, we numerically evaluate the minimal embedding solution (without any nodes) of (3.8) with various charge densities of D-instantons represented by $q$ and the results are illustrated as in Fig. 3. Our results show that $q=0$ corresponds to the minimal embedding among various

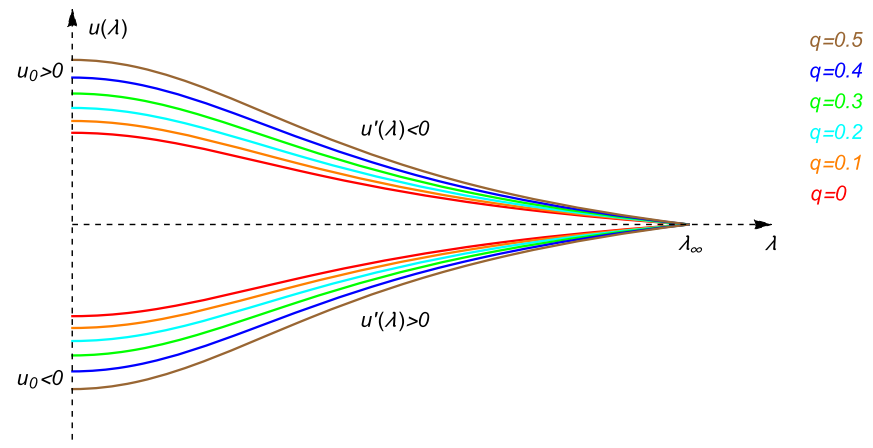

FIG. 3. The minimal embedding function $u(\lambda)$ of flavor brane with various $q$ and the parameter is set to be $\lambda_{\infty}=5.73 r_{\mathrm{KK}}$. 

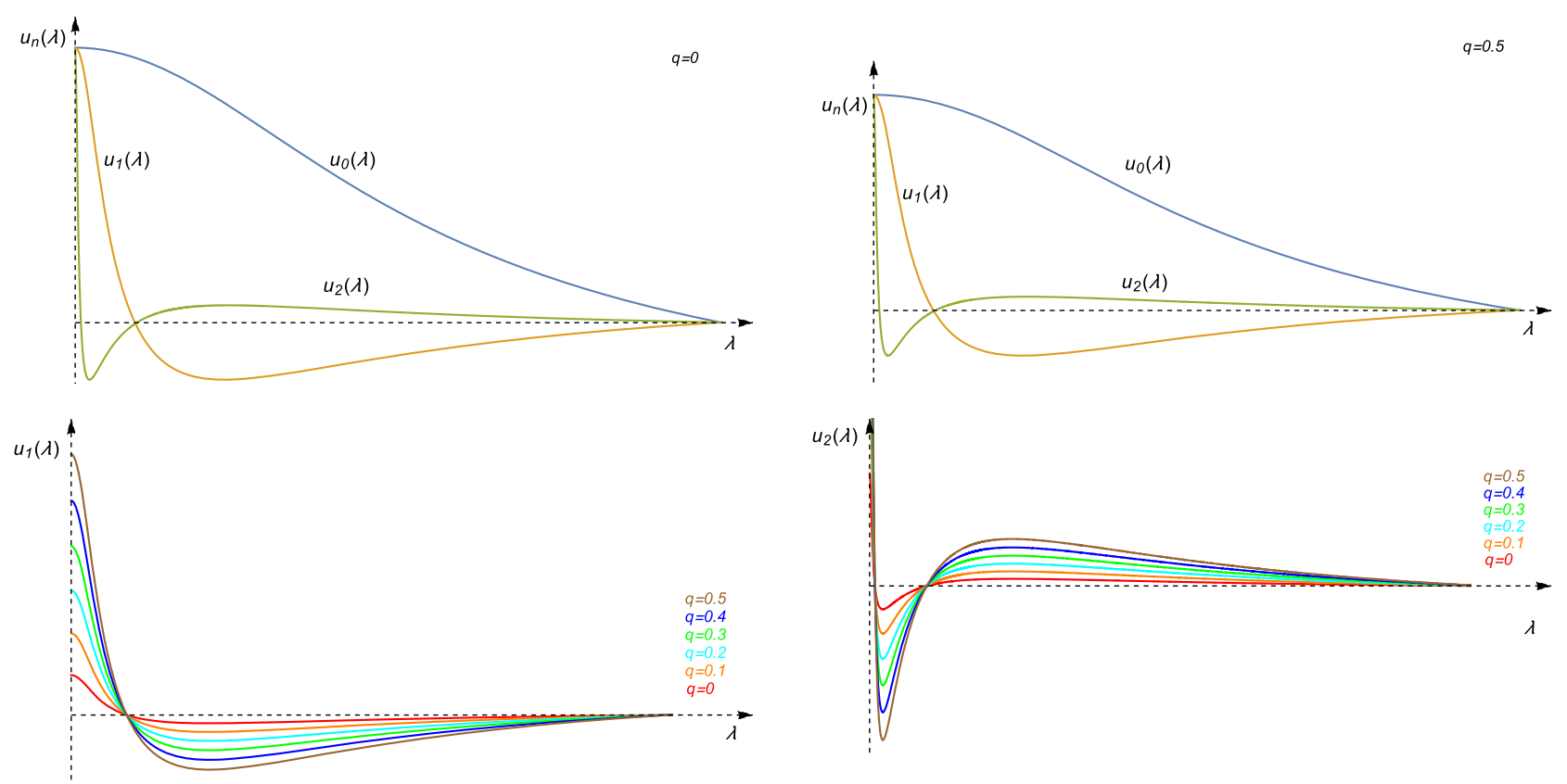

FIG. 4. The upper figures illustrate the relation of $u_{0,1,2}(\lambda)$ with $q=0,0.5$, and the lower figures show the relation of $u_{1,2}$ respective to various $q$.

values of $q$ respected to the solutions with zero node, and this is consistent with how the D3-D-instanton solution describes the dual theory in an excited background. Equation (3.8) also includes solutions with $n$ nodes, and let us denote it as $u_{n}(\lambda)$, so the minimal solutions are identified as $u_{0}(\lambda, q)$ now. We also show the numerical relation of $u_{n=0,1,2}(\lambda, q)$ for a fixed $q$ and $u_{1,2}(\lambda, q)$ with various $q$ as in Fig. 4 . The numerical calculation implies that the associated energy of the embedded flavor brane is a monotonically increased function of the number of nodes for any $q$, and this is numerically verified as in Fig. 5. In this sense, the solution of maximal embedding presented in (3.11) can be treated as having infinity nodes. On the other hand, we can find the total energy of a flavor brane is always minimized at $q=0$. To verify this conclusion

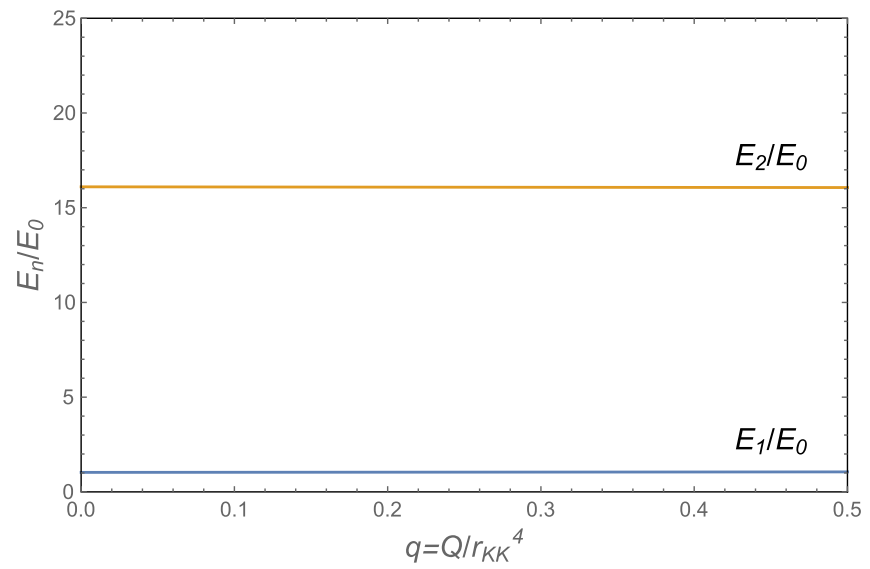

FIG. 5. The ratios of $E_{n}$ and $E_{0} . E_{n}$ refers to the energy of a flavor brane with $n$-nodes embedding function $u_{n}(\lambda)$. quantitatively, we could in particular evaluate the energy density of a maximal embedded flavor brane since it would be semianalytical. Plugging (3.11) into (3.6), we could obtain the energy density of a maximal embedded flavor brane as

$E_{\mathrm{D} 7}^{\max }=-\frac{1}{V_{3}} S_{\mathrm{D} 7}^{\max }=T_{\mathrm{D} 7} V_{S^{4}} R^{2}\left[\frac{\lambda_{\infty}}{3}+b_{\max }(q) r_{\mathrm{KK}}^{3}\right]$.

Here $b_{\max }(q)$ is a constant dependent on $q$ that can be expressed by the combination of generalized hypergeometrical functions. We plot out the numerical values of $b_{\max }(q)$ as in Fig. 6, and it indeed shows $q=0$ corresponds

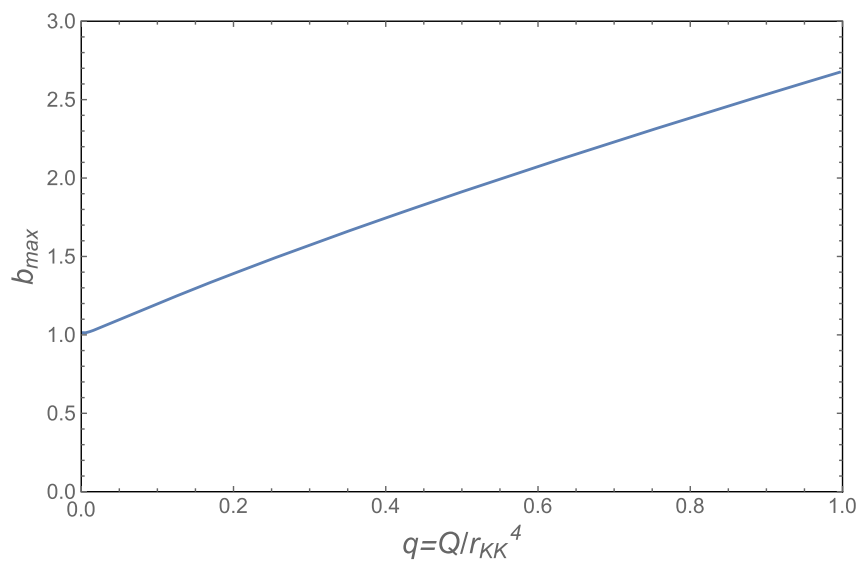

FIG. 6. The relation of $b_{\max }$ and $q$. The numerical value shows $b_{\max }(0) \simeq 1.026$ which exactly returns to the result in [15]. 
to the flavor brane of the lowest energy. In this sense, the vacuum with nonzero $q$ could be recognized as the metastable vacua of flavors in the presence of instantons in the dual theory, which is in agreement with $[14,15]$.

Since we are usually interested in comparing energies with the same boundary condition between different solutions, the maximal energy (3.17) should be subtracted as the regularization of the total energy. Let us denote the on-shell action with $n$-nodes embedding function $u_{n}(\lambda)$ as $S_{\mathrm{D} 7}^{n}$, so the total energy of the flavor brane is redefined as

$$
E_{f}^{n}(q)=-\frac{1}{V_{3}}\left(S_{\mathrm{D} 7}^{n}-S_{\mathrm{D} 7}^{\max }\right) .
$$

For the minimal embeddings of two parity-related flavor branes, Eq. (3.18) could be evaluated as

$$
E_{f}^{0}(q) \simeq-N_{c}\left(g_{s} N_{c}\right)\left[b_{0}(q) M_{\mathrm{KK}}^{3}+a_{0}(q) M_{\mu}^{3}\right],
$$

where $b_{0}(q)$ and $a_{0}(q)$ depend on the D-instanton charge $q$. The energy scale $M_{\mu}$ is related to the length scale $\mu=$ $M_{\mu} R^{2} / 2$, which comes from the duality of holographic radius/energy relation [44]. The difference between the energy density at large $N_{c}$ should be relevant to the potential barrier of the instanton vacuum in the dual theory. Besides, the general configuration of $N_{f}$ flavor branes can also be obtained by (3.19). Let us consider $p$ of $N_{f}$ flavor branes located in the upper $u, \lambda$ plane while the other $N_{f}-p$ flavor branes located the lower plane with minimal embedding. Since the energy of each flavor brane is equivalent, the total energy of these flavor branes should be

$E_{f, \mathrm{tot}}^{0}=p E_{f}^{0}(q)+\left(N_{f}-p\right) E_{f}^{0}(q)=N_{f} E_{f}^{0}(q)$.

\section{Massive case}

Let us turn to the massive case by considering the inclusion of a bare mass of the quarks or fermions in the dual theory. The bare mass in this model can be viewed as a source for the condensate operator $\langle\bar{\psi} \psi\rangle$ of fermions. Since the bare mass of fermions is identified as the spatial separation between D3- and D7-branes along the transverse direction $u$ in the UV region and we have seen in the last subsection

$$
u(\lambda) \rightarrow \frac{1}{\sqrt{\lambda}},
$$

at large $\lambda$, we can set

$$
\lim _{\lambda \rightarrow \lambda_{\infty}} \sqrt{\frac{\lambda}{\mu}} u(\lambda)=2 \pi l_{s}^{2} m .
$$

This is also equivalent to set $u_{\infty} \simeq 2 \pi l_{s}^{2} m$ while this boundary condition breaks the parity symmetry. So the parity transformation $u \rightarrow-u$ implies the flavor branes with up/down embedding have to take a positive/negative mass which succeeds the discussion in the D3/D7 approach consistently. It means the fermion mass leads to the degeneracy between the upper and lower embedding of the flavor branes. Then in order to obtain the energy of the flavor brane in the massive case, we can start from the massless case by performing a small variation in the boundary condition,

$$
\delta u_{\infty}=\sqrt{\frac{\mu}{\lambda_{\infty}}} 2 \pi l_{s}^{2} \delta m .
$$

So the associated variation in the on-shell action of the flavor brane becomes

$$
\begin{aligned}
\delta S_{\mathrm{D} 7}= & \left.\frac{\partial \mathcal{L}}{\partial u^{\prime}} \delta u\right|_{\lambda=0} ^{\lambda=\lambda_{\infty}}=-T_{\mathrm{D} 7} V_{3} V_{S^{4}} R^{2}\left[e^{\phi}\left(r_{\mathrm{KK}}^{4}+4 \rho^{4}\right)^{3 / 2}\right. \\
& \left.\times \frac{\lambda^{4}}{8 \rho^{8}} \frac{u^{\prime}}{\sqrt{1+u^{\prime 2}}} \delta u\right]\left.\right|_{\lambda=0} ^{\lambda=\lambda_{\infty}}
\end{aligned}
$$

which leads to

$$
\begin{aligned}
\delta E_{f} & =T_{\mathrm{D} 7} V_{S^{4}} R^{2} \lambda_{\infty}^{2} u^{\prime}\left(\lambda_{\infty}\right) \delta u_{\infty} e^{\phi\left(\lambda_{\infty}\right)} \equiv \mp c\left(q, \lambda_{\infty}\right) \delta m, \\
c\left(q, \lambda_{\infty}\right) & =e^{\phi\left(\lambda_{\infty}\right)} \frac{N_{c} \sqrt{g_{s} N_{c}}}{24 \pi^{5 / 2}} M_{\mu}^{2},
\end{aligned}
$$

where we have used $u^{\prime}(0)=0$ and the equation of motion for $u(\lambda)$. Since the embedding function can have both signs as in the massless case characterized by $u_{\infty}^{\prime}$, the positive/ negative mass $m$ determines the sign of $u_{\infty}^{\prime}$ as it is preferred. As the massless case, the energy of the flavor brane can be obtained by using (3.18) which consists of the massless part $E_{f}^{0}(q)$ plus a small variation $\delta E_{f}$ as

$$
\begin{aligned}
E_{f}^{0}(q, m) & =E_{f}^{0}(q)+\delta E_{f} \\
& \simeq E_{f}^{0}(q) \mp c\left(q, \lambda_{\infty}\right) m .
\end{aligned}
$$

This result shows that the fermion condensate is negative/ positive for positive/negative mass due to

$$
\langle\bar{\psi} \psi\rangle=\frac{d E_{f}^{0}(q, m)}{d m}=\mp c\left(q, \lambda_{\infty}\right) \operatorname{sign}(m) .
$$

And it would be slightly modified by the presence of the D-instantons in the quadratic order of the fermion mass.

To close this subsection, let us evaluate the total energy of $N_{f}$ flavor branes with a bare mass. As before, we consider the configuration that $p$ of $N_{f}$ branes wraps the upper half-five-sphere separated from the other $N_{f}-p$ branes wrapping the lower half-five-sphere with a common mass $m$. So the total energy is a collection of upper and lower branes which is 


$$
\begin{aligned}
E_{f, \mathrm{tot}}^{0}(q, m)= & p\left[E_{f}^{0}(q)-c\left(q, \lambda_{\infty}\right) m\right] \\
& +\left(N_{f}-p\right)\left[E_{f}^{0}(q)+c\left(q, \lambda_{\infty}\right) m\right] \\
= & N_{f} E_{f}^{0}(q)+\left(N_{f}-2 p\right) c\left(q, \lambda_{\infty}\right) m .
\end{aligned}
$$

It would be obvious that, for any $q$, the minimal energy occurs at $p=N_{f}$ for $m>0$ and $p=0$ for $m<0$. And (3.28) reduces to (3.20) if $m \rightarrow 0$ so that the degeneracy of upper and lower embeddings is regained.

\section{B. Embedding of the CS D7-brane}

Since the R-R flux $C_{0}$ is nonvanished in our D3-D(-1) background, there should also be a magnetic source for $C_{0}$. And the source could be provided by $n_{b}$ CS D7-branes as probes coupled to $C_{0}$ magnetically. The configuration of the CS brane is illustrated in Table I. Since the number of CS branes should be an integer, the CS level is automatically quantized in holography.

In the D3-brane approach, the CS brane can be set located at $r=r_{\mathrm{KK}}$ in order to minimize their energy density; however, this does not work in the presence of D-instantons because the energy density of a single CS brane is evaluated as

$$
\begin{aligned}
S_{\mathrm{D} 7}^{\mathrm{CS}} & =-T_{\mathrm{D} 7} \int d^{8} x e^{-\phi} \sqrt{-g_{\mathrm{D} 7}}-\mu_{7} \int C_{8} \\
& =-T_{\mathrm{D} 7} V_{3} V_{S^{5}} R^{2} r^{3} e^{\phi}-N_{\mathrm{D}},
\end{aligned}
$$

where $C_{8}$ is the dual form of $C_{0}$ defined as $d C_{8}={ }^{\star} d C_{0}$. This action is divergent at $r=r_{\mathrm{KK}}$ which leads to an IR divergence in the dual field. While this is not important when we are interested in comparing the difference of the energy, the position of the CS brane would be less clear. To figure out this problem we require that our discussion should reduce to the D3-brane approach if $q \rightarrow 0$. In this sense, we assume that the location of the CS brane $r=r_{\mathrm{KK}}$ is shifted by the presence of D-instantons which becomes $r=r_{Q}>r_{\mathrm{KK}}$. And we furthermore treat the solution (3.11)

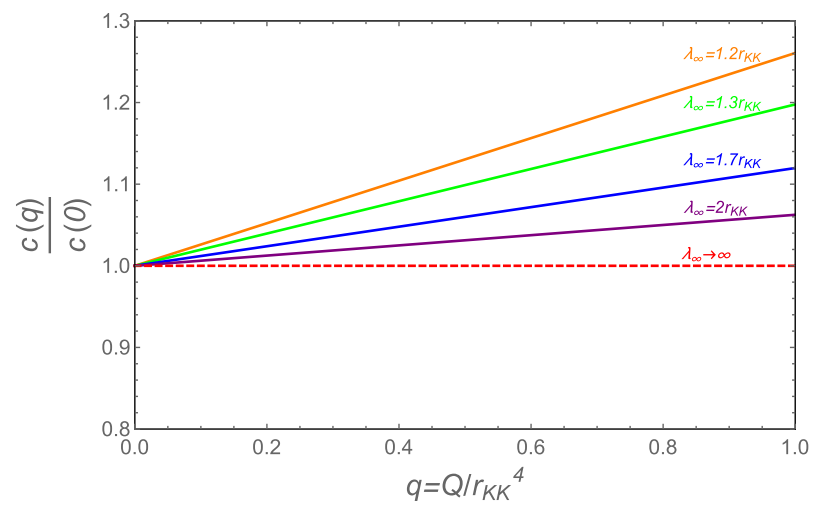

describing a CS D7-brane embedding at $r=r_{Q}$ according to the embedding equation (3.8), so that

$$
r_{Q}=\frac{1}{\sqrt{2}}\left(k+\frac{1}{k}\right)^{1 / 2} r_{\mathrm{KK}} .
$$

Hence for a fixed $q$, the maximal embedding of a flavor D7-brane can be identified as an embedding function of CS D7-brane as it is done in the D3-brane approach. The positive and negative level of the CS brane corresponds to the orientation of counterclockwise and clockwise, respectively, in the $u, \lambda$ plane. Therefore the total energy of a CS brane can be evaluated as

$$
\begin{aligned}
E_{\mathrm{CS}}(q) & =-\frac{S_{\mathrm{D} 7}^{\mathrm{CS}}}{V_{3}}=\mu_{7} V_{S^{5}} R^{2} r_{Q}^{3} e^{\phi\left(r_{Q}\right)} \\
& =\frac{g_{s} N_{c}^{2} M_{\mathrm{KK}}^{3}}{64 \pi^{2}}\left[\frac{1}{2 \sqrt{2}}\left(k+\frac{1}{k}\right)^{3 / 2} e^{\phi\left(r_{Q}\right)}+\frac{1}{2} q\right],
\end{aligned}
$$

which indeed reduces to the D3-brane approach when $q \rightarrow 0$. We plot out $G(q)=E_{\mathrm{CS}}(q) / E_{\mathrm{CS}}(0)$ and $c\left(q, \lambda_{\infty}\right) /$ $c\left(0, \lambda_{\infty}\right)$ as a function of $q$ in Fig. 7. The numerical result also illustrates $q=0$ and corresponds to the CS brane with minimal energy and the limit of $r_{Q} \rightarrow r_{\mathrm{KK}}$.

At low energy, the CS brane reduces to a 3D $U\left(n_{b}\right)$ gauge theory and leads to a Chern-Simons action at level $-N$ due to the Wess-Zumino term of the D-brane action which is

$$
\begin{aligned}
S_{C_{4}} & =\frac{1}{2(2 \pi)^{5} l_{s}^{4}} \int_{\mathrm{D} 7} C_{4} \wedge \operatorname{Tr}(F \wedge F) \\
& =-\frac{1}{2(2 \pi)^{5} l_{s}^{4}} \int_{S^{5}} F_{5} \int_{\mathbb{R}^{1,2}} \operatorname{Tr} \omega_{3}=-\frac{N_{c}}{4 \pi} \int_{\mathbb{R}^{1,2}} \operatorname{Tr} \omega_{3} .
\end{aligned}
$$

All excitations on the CS branes will decouple at very low energy scale except this CS term, so we can obtain the

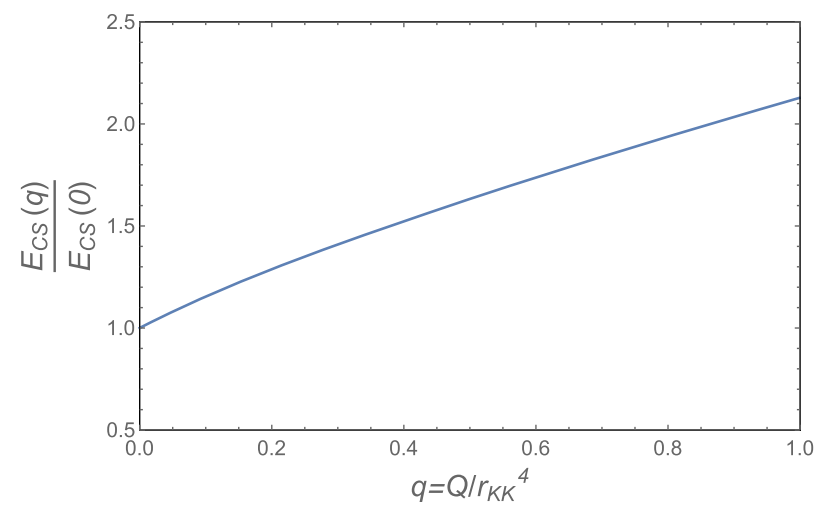

FIG. 7. The relation of $c\left(q, \lambda_{\infty}\right)$ (left), $G(q)=E_{\mathrm{CS}}(q) / E_{\mathrm{CS}}(0)$ (right), and $q$. For $q=0$, it returns to the D3-brane approach without any D-instantons. 
level/rank duality $S U(N)_{n_{b}} \leftrightarrow U\left(\left|n_{b}\right|\right)_{N}$ through this holographic system as the quantum field theory expectations.

\section{VACUUM STRUCTURE OF THE DUAL THEORY}

In this section, let us analyze the vacuum structure in the dual theory at the large- $N_{c}$ expansion. Since the vacuum of the dual theory in general may include both flavor and CS branes, we are going to take into account the configuration with two kinds of the D7-branes.

The effective CS level in the dual theory is required as

$$
\left.i \int_{S^{1}} F_{1}\right|_{r \rightarrow \infty}=k_{\mathrm{eff}} \text {. }
$$

However, in order to define the CS level $\kappa=k_{b}-N_{f} / 2$, the CS level must depend on $p$. To find the result, we can straightforwardly count the number of the orientation in the $u, \lambda$ plane. Defining the number of D7-branes with counterclockwise/clockwise orientation is positive/negative; let us consider the configuration of that, in the $u, \lambda$ plane $k_{0}$ counterclockwise CS branes live in $\mathcal{R}_{0}, p$ flavor branes live in $\mathcal{R}_{+}$, and $N_{f}-p$ flavor branes live in $\mathcal{R}_{-}$ where $\mathcal{R}_{+}, \mathcal{R}_{-}, \mathcal{R}_{0}$, respectively, refers to the regions of the $u, \lambda$ plane which are above, between, and below the flavor branes as it is illustrated in Fig. 8. We only consider the minimal embedding of the flavor branes since the concern is the vacuum structure. Requiring $\kappa=k_{\text {eff }}$ at the UV boundary, we have [15]

$$
k_{\mathrm{eff}}= \begin{cases}\kappa-\frac{N_{f}}{2}, & \text { in } \mathcal{R}_{+}, \\ \kappa+p-\frac{N_{f}}{2}, & \text { in } \mathcal{R}_{0}, \\ \kappa+\frac{N_{f}}{2}, & \text { in } \mathcal{R}_{-},\end{cases}
$$

and $k_{0}=\kappa+p-N_{f} / 2$ which is what we desire in the dual field theory. The interpretation of such a D-brane configuration at low energy is that the flavor symmetry $U\left(N_{f}\right)$ is broken spontaneously to $U(p) \times U\left(N_{f}-p\right)$,

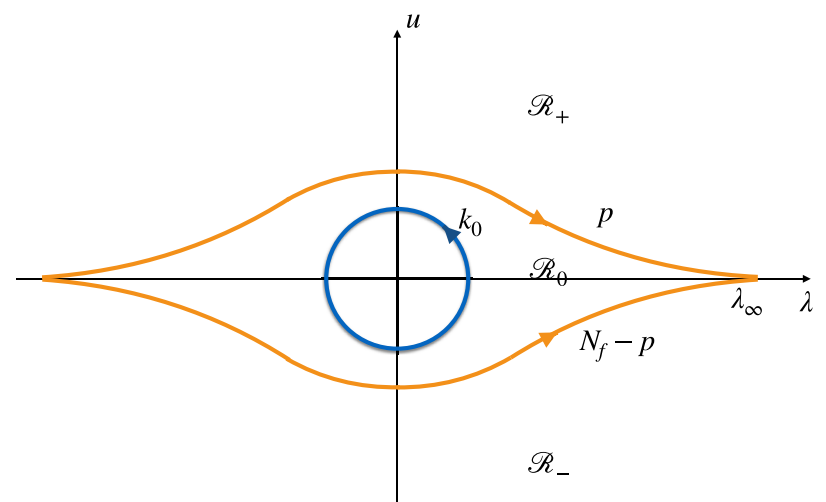

FIG. 8. The configuration of flavor and CS branes on the $u, \lambda$ plane. The flavor branes are represented by the orange lines, and the CS branes are represented by the blue line. $\mathcal{R}_{+}, \mathcal{R}_{-}, \mathcal{R}_{0}$, respectively, refers to the regions above, between, and below the flavor branes.

which creates $2 p\left(N_{f}-p\right)$ Goldstone bosons and their target space is Grassmann,

$$
\operatorname{Gr}\left(p, N_{f}\right)=\frac{U\left(N_{f}\right)}{U(p) \times U\left(N_{f}-p\right)} .
$$

The CS branes leads to a level/rank duality of $U(\mid \kappa+p-$ $\left.N_{f} / 2 \mid\right)_{N} \leftrightarrow S U(N)_{k+p-N_{f} / 2}$. So the low-energy dynamics of a $p$ sector would have the symmetry

$$
\operatorname{Gr}\left(p, N_{f}\right) \times S U(N)_{k+p-N_{f} / 2},
$$

in which the $N_{f}+1$ sectors describe the vacuum of the dual theory holographically. To analyze the phase structure of the vacuum, the minimal value of the (free) energy is necessary. Since the total energy of the $p$ sector consists of flavor plus the CS part and the flavor energy has be obtained in (3.28), we need to include the energy of the CS brane, which is the number of CS branes times the energy density $E_{\mathrm{CS}}(q)$ given in (3.31). Therefore the total free energy density is collected as $\left(\kappa \geq 0,0 \leq p \leq N_{f}\right)[15]$,

$$
E(p, q)=N_{f} E_{f}^{0}(q)+\left(N_{f}-2 p\right) c\left(q, \lambda_{\infty}\right) m+\left|\kappa+p-N_{f} / 2\right| E_{\mathrm{CS}} .
$$

Minimize (4.5) and then compare the free energy; the associated free energy density is obtained as, for $\kappa>N_{f} / 2$,

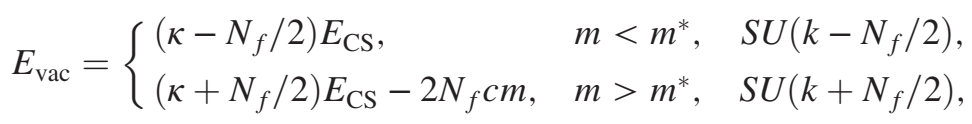

where $S U\left(k \pm N_{f} / 2\right)$ refers to the corresponding topological phase in the dual theory. And for $\kappa<N_{f} / 2$, the minimized free energy density and associated topological phase are collected as

$$
E_{\mathrm{vac}}=\left\{\begin{array}{llc}
\left(N_{f} / 2-\kappa\right) E_{\mathrm{CS}}, & m<-m^{*}, & S U\left(k-N_{f} / 2\right), \\
2\left(\kappa-N_{f} / 2\right) c\left(q, \lambda_{\infty}\right) m, & -m^{*}<m<m^{*}, & \operatorname{Gr}\left(p, N_{f}\right), \\
\left(N_{f} / 2+\kappa\right) E_{\mathrm{CS}}-2 N_{f} c\left(q, \lambda_{\infty}\right) m, & m>m^{*}, & S U\left(k+N_{f} / 2\right),
\end{array}\right.
$$




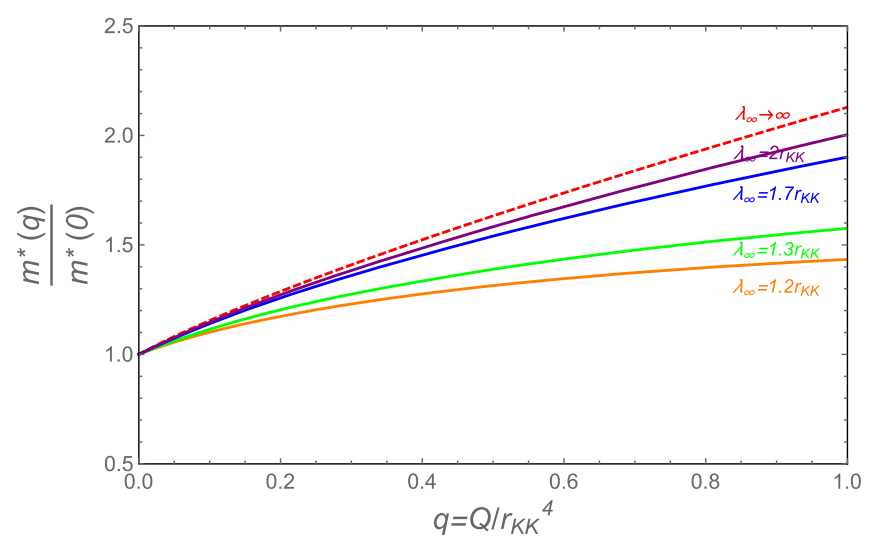

FIG. 9. Relation of $m^{*}(q)$ and $q$. The critical mass for the phase transition is increased by the presence of the D-instantons.

where the critical mass $m^{*}$ is defined as

$$
m^{*}=\frac{E_{\mathrm{CS}}(q)}{2 c\left(q, \lambda_{\infty}\right)} .
$$

Since $c\left(q, \lambda_{\infty}\right)$ never goes to zero, the derivative with respect to $m$ in both (4.6) and (4.7) is discontinuous, which means there remains to be a first order phase transition at $m= \pm m^{*}$ in the presence of the D-instantons. And the vacua would be degenerate at the critical point. However, in our holographic approach, the mass $m^{*}$ additionally depends on the charge density $q$ of the D-instantons. So we numerically evaluate $m^{*}$ as a function of $q$ as in Fig. 9. According to the numerical calculation, while the order parameter $c\left(q, \lambda_{\infty}\right)$ in the UV limit $\lambda_{\infty} \rightarrow 0$ is almost unchanged, the critical mass $m^{*}$ is increased by the presence of the D-instantons, which shifts the phase transition point for $\kappa>N_{f} / 2$, thus enhancing the phase of $\operatorname{Gr}\left(p, N_{f}\right)$ for $\kappa<N_{f} / 2$. Accordingly, the D3-D(-1) approach implies the phase transition point is also determined by the D-instanton charge. This could be interpreted as the topological effect in the dual theory, which is similar as the topological contribution to the mass in the presence of the CS term [30].

\section{ENTANGLEMENT ENTROPY AND CONFINEMENT}

Since the entanglement entropy is expected to be a tool to characterize the confinement/deconfinement phases of the dual theory [39-42], in this section we will compute the quantum entanglement entropy between two physically disjoint spatial regions in the bulk and then compare the results with the analysis of the free energy.

Before the holographic calculation, we first take into account the simplest geometry: region $A$ is the product of $\mathbb{R}^{2} \times I_{l}$ where $I_{l}$ is a line interval of length $l$ and region $B$ is the complement of $A$. According to the AdS/CFT dictionary, the quantum entanglement entropy between regions $A$ and $B$ relates to the surface $\gamma$ in bulk whose boundary coincides with the boundary of $A$. Supposing we are discussing the correspondence of $\mathrm{AdS}_{\mathrm{d}+2} / \mathrm{CFT}_{\mathrm{d}+1}$, the classical area of surface $\gamma$ is given as

$$
S_{\gamma}=\frac{1}{4 G_{N}^{d+2}} \int_{\gamma} d^{d} x \sqrt{g_{\text {ind }}},
$$

where $G_{N}^{d+2}$ is the $(d+2)$-dimensional Newton constant and $g_{\text {ind }}$ refers to the induced metric on $\gamma$. Notice $\gamma$ has to be spatial-like to represent the entanglement entropy at a fixed time. The (5.1) can also be generalized into nonconformal situations. For example, in 10D geometry of D-branes, Eq. (5.1) could be naturally modified as

$$
S_{A}=\frac{1}{4 G_{N}^{10}} \int_{\gamma} d^{8} x \sqrt{g_{\text {ind }}} .
$$

We will use (5.2) to evaluate the quantum entanglement entropy in our holographic model.

The most convenient way to begin the calculation is to write the $10 \mathrm{D}$ metric as

$d s^{2}=\alpha(r)\left[\beta(r) d r^{2}+\eta_{\mu \nu} d x^{\mu} d x^{\nu}\right]+g_{m n} d y^{m} d y^{n}$,

where $\mu=0,1, \ldots, d, m=d+2, \ldots, 9$ parametrize $\mathbb{R}^{d+1}$ and $8-d$ internal directions, respectively, and $r$ refers to the holographic radial coordinate. Using (5.2) with formula (5.3), the minimized action is given as

$$
S_{A}=\frac{V_{d-1}}{2 G_{N}^{10}} \int_{r_{*}}^{r_{\infty}} d r \frac{\sqrt{\beta(r)} H(r)}{\sqrt{H(r)-H\left(r_{*}\right)}},
$$

where

$$
\begin{aligned}
l\left(r_{*}\right) & =2 \sqrt{H\left(r_{*}\right)} \int_{r_{*}}^{\infty} d r \frac{\sqrt{\beta(r)}}{\sqrt{H(r)-H\left(r_{*}\right)}}, \\
H(r) & =e^{-4 \phi} V_{\mathrm{int}}^{2} \alpha^{d}(r) \\
V_{\mathrm{int}} & =\int \prod_{m=1}^{8-d} d y^{m} \sqrt{\operatorname{det} g} .
\end{aligned}
$$

The minimal surface has distinct features for small and large $l$ according to the definition of regions $A$ and $B$. The minimal surface extends into the bulk up to the radial position $r_{*}>r_{\mathrm{KK}}$ as a connected surface for small $l$ while the minimal surface becomes two disconnected pieces and extends in the bulk all the way up to $r_{\mathrm{KK}}$ for large $l$. In order to characterize the phase transition, we need to compare the entanglement entropy of the connected with the disconnected configuration of the minimal surface. While the entanglement entropy itself may be divergent, its difference $\Delta S$ could be finite, which according to (5.4) could be written as 


$$
\begin{aligned}
\Delta S \equiv & \frac{2 G_{N}^{10}}{V_{d-1}}\left(S_{A}^{\mathrm{conf}}-S_{A}^{\mathrm{dic}}\right) \\
= & \int_{r_{*}}^{\infty} d r \sqrt{\beta(r) H(r)}\left\{\left[1-\frac{H\left(r_{*}\right)}{H(r)}\right]^{-1 / 2}-1\right\} \\
& -\int_{r_{\mathrm{KK}}}^{r_{*}} d r \sqrt{\beta(r) H(r)} .
\end{aligned}
$$

Plugging (2.4) into (5.3)-(5.6), we can numerically calculate the relation of $\Delta S$ and $l, l$ and $r_{*}$ as illustrated in Fig. 10. As we can see, the critical length $l_{c}(\Delta S=0)$ is increased by the density of the D-instanton charge denoted by $q$, which implies there would be a first order phase transition at scale $l_{c}$ and it is enhanced in the presence of the D-instantons. Since the entanglement entropy takes the order of $\mathcal{O}\left(N_{c}^{0}\right)$ and $\mathcal{O}\left(N_{c}^{2}\right)$, respectively, for $l>l_{c}$ and $l<l_{c}$, this phase transition may relate to the deconfinement phase transition in a QCD-like theory. So it would be, on the other hand, very interesting to evaluate the critical temperature of the deconfinement phase transition to examine whether it is consistent with the analyses of the entanglement entropy in holography.

To obtain the critical temperature of the deconfinement in the dual theory, we should compute the associated free energy $F$ in holography, which is the summary of the Euclidean version of the on-shell action (2.1) denoted as $S_{\mathrm{IIB}}^{E}$, Gibbons-Hawking term $S_{\mathrm{GH}}$, and holographic counterterm $S_{\mathrm{ct}}^{\text {bulk }}$ in bulk, since the deconfinement phase transition is suggested to be identified as the Hawking-Page transition in the bulk [35-38]. In Einstein frame, they are given as $[45,46]$,

$$
\begin{aligned}
S_{\mathrm{bulk}} & =S_{\mathrm{IIB}}^{E}+S_{\mathrm{GH}}+S_{\mathrm{ct}}^{\text {bulk }}, \\
S_{\mathrm{IIB}}^{E} & =-\frac{1}{2 \kappa_{10}^{2}} \int d^{10} x \sqrt{g}\left[\mathcal{R}-\frac{1}{2} \partial \Phi \cdot \partial \Phi-\frac{1}{2} e^{2 \Phi}\left|F_{1}\right|^{2}-\frac{1}{2}\left|F_{5}\right|^{2}\right], \\
S_{\mathrm{GH}} & =-\frac{1}{\kappa_{10}^{2}} \int d^{9} x e^{-2 \Phi_{0}} \sqrt{h}\left(K-K_{0}\right), \\
S_{\mathrm{ct}}^{\text {bulk }} & =-\frac{1}{\kappa_{10}^{2}} \int d^{9} x e^{-2 \Phi_{0}} \frac{5}{R} \sqrt{h},
\end{aligned}
$$

where $h$ is the determinant of the boundary metric, i.e., the slice of the 10D metric (2.4) in the Einstein frame at fixed $r=r_{\infty}$ with $r_{\infty} \rightarrow \infty . K$ is the trace of the extrinsic curvature at the boundary, $K_{0}$ arises from the standard transformation of the gravity action from Einstein to string frame, and they are given as

$K=-\left.\frac{1}{\sqrt{g}} \partial_{r} \sqrt{h}\right|_{r \rightarrow \infty}, \quad K_{0}=\left.\frac{9}{4} \sqrt{g^{r r}} \partial_{r} \phi\right|_{r \rightarrow \infty}$.
Then to include the contribution of the flavors and CS level, we additionally need to evaluate the Euclidean on-shell action of the flavor and CS brane with respect to backgrounds (2.2) and (2.4). For the flavor D7-brane, it is embedded at $x^{3}, u=$ const, and the on-shell action and the holographic counterterm $S_{\mathrm{ct}}^{f}$ could be chosen as [46-48]
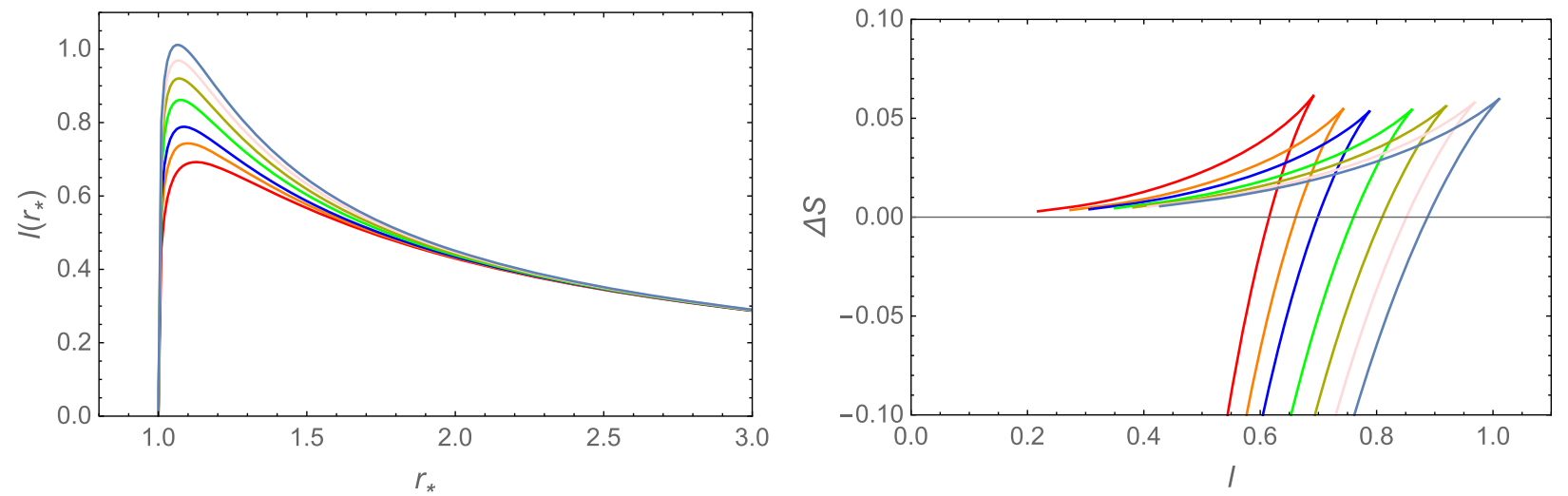

FIG. 10. Left: Relation of $l$ and $r_{*}$ for $q=0,0.5,1,2,3,4,5$ (lower to upper); $l_{\max }$ is increased by $q$. Right: Relation of $\Delta S$ and $l$ for $q=0,0.5,1,2,3,4,5$ (left to right) as a typical swallowtail behavior. The critical length $l_{c}$ is also increased by $q$. 


$$
\begin{aligned}
S_{f} & =S_{\mathrm{DBI}}+S_{\mathrm{ct}}^{f}, \\
S_{\mathrm{DBI}} & =-N_{f} T_{\mathrm{D} 7} \int d^{8} x e^{\phi} \sqrt{g}, \\
S_{\mathrm{ct}}^{f} & =\frac{R}{3} T_{\mathrm{D} 7} \int d^{7} x \sqrt{h_{\mathrm{D} 7}} .
\end{aligned}
$$

We note that the metric presented in (5.7)-(5.9) refers to the metrics (2.2) and (2.4) in the Einstein frame which is defined as $g_{\text {string }}=g_{\text {Einstein }} e^{\phi / 2}$. For the CS brane, it is embedded at $x^{3}=$ const and $r=r_{Q}, r_{H}$ with respect to (2.2) and (2.4). The on-shell action of a CS brane evaluated in the confining background has been given in (3.31) while it vanishes in the black brane background (2.2) calculated by using (5.9). Therefore the total on-shell action including the bulk part, flavor part, and CS part is

$$
S_{\text {on-shell }}=S_{\text {bulk }}+S_{f}+S_{\mathrm{CS}}
$$

Afterwards recalling the AdS/CFT dictionary,

$$
\left\langle e^{-F}\right\rangle=e^{S_{\text {on-shell }}}
$$

with the solutions (2.2) and (2.4) we can obtain the free energy $F$, respectively,

$$
\begin{aligned}
& F_{d}=-\frac{1}{8} N_{c}^{2} \pi^{2} T^{4} V_{3} \beta_{T}-\frac{36 \pi}{\lambda_{t}} N_{c} N_{\mathrm{D}}+\frac{1}{18} N_{c} N_{f} T^{3} V_{3} \lambda_{t}\left[(\pi+\ln 64) q_{T}-2\right], \\
& F_{c}=-\frac{M_{\mathrm{KK}}^{4} N_{c}^{2} V_{3} \beta_{3}}{128 \pi^{2}}-\frac{36 \pi}{\lambda_{t}} N_{c} N_{\mathrm{D}}+\frac{\lambda M_{\mathrm{KK}}^{3} N_{c} k_{0} V_{3}}{64 \pi^{2}} G(q)+\frac{\lambda_{t} M_{\mathrm{KK}}^{3} N_{c} N_{f} V_{3}[2+q(8+\pi-\ln 4)]}{512 \sqrt{2} \pi^{3 / 2} \Gamma\left(\frac{7}{4}\right)^{2}}, \\
& \beta_{T}=1 / T, \quad q_{T}=Q / r_{H}^{4}, \quad \beta_{3}=2 \pi / M_{\mathrm{KK}},
\end{aligned}
$$

where $\lambda_{t}$ is the 't Hooft coupling constant defined as $\lambda_{t}=g_{s} N_{c}$ and $F_{d, c}$ refers to the free energy evaluated in the backgrounds (2.2) and (2.4), respectively. $k_{0}$ is the number of CS branes, and we have assumed $k_{0}$ and $N_{f}$ take the same order at large- $N_{c}$ due to the flavor and CS branes as probes. $G(q)$ is a function defined as $G(q)=E_{\mathrm{CS}}(q) /$ $E_{\mathrm{CS}}(0)$ whose behavior has been numerically illustrated in Fig. 7. Following the most discussion in gauge/gravity duality [35-38], the black brane (2.2) and soliton (bubble) solution (2.4), respectively, correspond to the deconfinement and confinement phases in the dual theory, so the phase transition can be obtained by comparing their free energy, which identifies the confinement/deconfinement phase transition in the field theory as the Hawking-Page transition in the bulk. According to (5.12), we can find the D-instantons as D(-1)-branes negatively increase the bulk free energy as a contribution of $\mathcal{O}\left(N_{c}^{2}\right)$ because at the large- $N_{c}$ limit, $N_{d} / N_{c}$ must be fixed; otherwise, the backreaction of the D-instantons in bulk vanishes. The critical temperature $T_{c}$ of the phase transition can be obtained by comparing the free energy at $F_{d}=F_{c}$, which is evaluated as

$$
\begin{aligned}
T_{c}= & \frac{M_{\mathrm{KK}}}{2 \pi}-\frac{\lambda_{t} M_{\mathrm{KK}}}{6 \pi^{2}} \frac{k_{0}}{N_{c}} G(q) \\
& -\frac{\lambda_{t} M_{\mathrm{KK}}}{432 \pi^{2}} \frac{N_{f}}{N_{c}}\left(C_{1}+C_{2} q\right)+\mathcal{O}\left(N_{c}^{-2}\right),
\end{aligned}
$$

where $C_{1,2}$ are two constants given as

$$
\begin{aligned}
C_{1} & =64 \pi+\frac{9 \sqrt{2} \pi^{5 / 2}}{\Gamma\left(\frac{7}{4}\right)^{2}} \simeq 464.66 \\
C_{2} & =\frac{9 \sqrt{2} \pi^{5 / 2}(8+\pi-\ln 4)}{2 \Gamma\left(\frac{7}{4}\right)^{2}}-32 \pi(\pi+\ln 64) \simeq 551.815 \\
q & =\frac{Q}{r_{\mathrm{KK}}^{4}}=\frac{128 \pi^{2}}{\lambda_{t} M_{\mathrm{KK}}^{3} V_{3}} \frac{N_{\mathrm{D}}}{N_{c}}
\end{aligned}
$$

Notice in the large- $N_{c}$ limit, $q$ is fixed and thus $G(q)$ is also fixed. So the critical temperature is not affected at $\mathcal{O}\left(N_{c}^{0}\right)$ while it decreases at $\mathcal{O}\left(N_{c}^{-1}\right)$ by the presence of the D-instantons through the flavor and CS branes due to $G(q)>0$. Since the behavior of $T_{c}$ is qualitatively consistent with the behavior of $l_{c} \sim T^{-1}$ obtained by evaluating the entanglement entropy, we may conclude that the entanglement entropy is indeed able to characterize the deconfinement phase transition.

\section{SUMMARY AND DISCUSSION}

In this work, by compactifying on the supersymmetry breaking $S^{1}$, we construct the supergravity solution for $N_{c}$ black D3-branes with dynamical $N_{\mathrm{D}}$ D-instantons, i.e., $\mathrm{D}(-1)$-branes, to obtain a 3D confining Yang-Mills in holography. To exhibit flavors and the CS term in the dual theory, we also add flavor and CS branes as a probe into the bulk geometry; hence the dual theory is expected to be a 3D YMCS with matters or CS QCD-like theory. The lowenergy regime of the 3D dual theory is analyzed by the IIB 
supergravity solution which geometrically shows the spontaneous breaking of the chiral symmetry $U\left(N_{f}\right)$ down to $U(p) \times U\left(N_{f}-p\right), p \in \mathbb{Z}$. And at very low energy, D-instantons could reduce to a pure CS theory. Because of the presence of the dynamical D-instantons, the embedding function of the flavor branes depends on the nonzero charge density of the D-instantons, which is realized to be metastable vacua of instantons in the dual theory. Then we further evaluate the vacuum structure of the dual theory by including both flavor and CS branes, which leads to a topological phase transition determined by the order parameter $m^{*}$ in the large- $N_{c}$ limit and $m^{*}$ is increased by the presence of D-instantons as it is expected. This behavior of $m^{*}$ can be interpreted as the topological contribution from the CS term in the dual theory, similarly as the topological contribution to mass in the CS theory. Moreover, we additionally evaluate the entanglement entropy and total free energy in holography to investigate the critical length $l_{c}$ and critical temperature $T_{c}$, which is expected to be the characters of the deconfinement phase transition. The behavior of $T_{c}$ is in qualitative agreement with the behavior of $l_{c}$ which implies the quantum entanglement entropy could indeed be a tool to determine confinement/deconfinement in this holographic approach.

We would like to give some comments to close this work. First, we notice that the discrepancy between topological phases characterized by $m^{*}$ becomes vanished if $E_{\mathrm{CS}} \rightarrow 0$. And in the black brane background (2.2), the CS brane is expected to be embedded at $r=r_{H}$ to minimize its energy which leads to a vanished $E_{\mathrm{CS}}$. Since the black brane background corresponds to a dual theory at finite temperature, the topological structure of the vacuum may therefore become vanished. So in this sense, our model might provide a holographic interpretation of why the topological aspects of hot QCD by instantons is quite difficult to be measured in experiment [49-52].

Second, it is expected that the topological phase transition is second order [1-7] if the number of the CS brane is
$\mathcal{O}\left(N_{c}\right)$. This can be achieved by taking into account the backreaction of CS branes. However, the number of CS branes is given by $\int_{S^{1}} F_{1}$ which relates to the boundary value of $C_{0}$ in our current setup. So the bulk dynamic could not involve the backreaction of CS branes in this work. The valid way to include the backreaction of CS branes is to solve the IIB supergravity action with a fluctuation of $C_{0}$ sourced by the CS branes, and then the next-to-leading-order contribution in the large- $N_{c}$ limit to the vacuum structure would be able to analyze in this sense. However, we would like to leave this for future study.

Last but not least, since the topological entanglement entropy is defined as the finite part of the entanglement $[53,54]$ which could be the measure of the topological order, $\Delta S$ should relate to the topological entanglement entropy. So the critical length $l_{c}$ seemingly shows the transition between the phases with different topological entanglement entropy. Thus if the entanglement entropy can characterize the deconfinement phase transition, $T_{c}$ may also reflect some properties of the topological order in the theory. However, our result also shows, in the large- $N_{c}$ limit, $T_{c}$ becomes nearly independent on the instantons while the behavior of $l_{c}$ remains to be determined by the instantons. Accordingly it seems the entanglement entropy is more sensitive to the topological properties of the theory than the critical temperature. And we expect it could be an instructive way to study the topological structure of YMCS theory.

\section{ACKNOWLEDGMENTS}

This work is supported by the National Natural Science Foundation of China (NSFC) under Grant No. 12005033, the research startup foundation of Dalian Maritime University in 2019 under Grant No. 02502608, and the Fundamental Research Funds for the Central Universities under Grant No. 3132021205.
[1] S. Giombi, S. Minwalla, S. Prakash, S. P. Trivedi, S. R. Wadia, and X. Yin, Chern-Simons theory with vector fermion matter, Eur. Phys. J. C 72, 2112 (2012).

[2] O. Aharony, G. Gur-Ari, and R. Yacoby, $d=3$ bosonic vector models coupled to Chern- Simons gauge theories, J. High Energy Phys. 03 (2012) 037.

[3] C.-M. Chang, S. Minwalla, T. Sharma, and X. Yin, ABJ triality: From higher spin fields to strings, J. Phys. A 46, 214009 (2013).

[4] S. Jain, S. P. Trivedi, S. R. Wadia, and S. Yokoyama, Supersymmetric Chern-Simons theories with vector matter, J. High Energy Phys. 10 (2012) 194.
[5] O. Aharony, G. Gur-Ari, and R. Yacoby, Correlation functions of large $\mathrm{N}$ chern- simons-matter theories and bosonization in three dimensions, J. High Energy Phys. 12 (2012) 028.

[6] G. Gur-Ari and R. Yacoby, Correlators of large N fermionic Chern-Simons vector models, J. High Energy Phys. 02 (2013) 150.

[7] S. Jain, S. Minwalla, and S. Yokoyama, Chern Simons duality with a fundamental boson and fermion, J. High Energy Phys. 11 (2013) 037.

[8] O. Aharony, Baryons, monopoles and dualities in ChernSimons-matter theories, J. High Energy Phys. 02 (2016) 093. 
[9] P. S. Hsin and N. Seiberg, Level/rank duality and ChernSimons-Matter theories, J. High Energy Phys. 09 (2016) 095.

[10] O. Aharony, S. S. Gubser, J. M. Maldacena, H. Ooguri, and Y. Oz, Large $\mathrm{N}$ field theories, string theory and gravity, Phys. Rep. 323, 183 (2000).

[11] E. Witten, Anti-de Sitter space and holography, Adv. Theor. Math. Phys. 2, 253 (1998).

[12] K. Becker, M. Becker, and J. H. Schwarz, String Theory and M-Theory, A Modern Introduction (Cambridge University Press, Cambridge, England, 2007).

[13] E. Witten, Anti-de Sitter space, thermal phase transition, and confinement in gauge theories, Adv. Theor. Math. Phys. 2, 505 (1998).

[14] D. K. Hong and H. U. Yee, Holographic aspects of three dimensional QCD from string theory, J. High Energy Phys. 05 (2010) 036; Erratum, J. High Energy Phys. 08 (2010) 120.

[15] R. Argurio, A. Armoni, M. Bertolini, F. Mignosa, and P. Niro, Vacuum structure of large $N \mathrm{QCD}_{3}$ from holography, J. High Energy Phys. 07 (2020) 134.

[16] M. Fujita, W. Li, S. Ryu, and T. Takayanagi, Fractional quantum Hall effect via holography: Chern-Simons, edge states, and hierarchy, J. High Energy Phys. 06 (2009) 066.

[17] T. Schäfer and E. V. Shuryak, Instantons in QCD, Rev. Mod. Phys. 70, 323 (1998).

[18] D. J. Gross, R. D. Pisarski, and L. G. Yaffe, QCD and instantons at finite temperature, Rev. Mod. Phys. 53, 43 (1981).

[19] E. Witten, Small instantons in string theory, Nucl. Phys. B460, 541 (1996).

[20] M. R. Douglas, Branes within branes, NATO Sci. Ser. C 520, 267 (1999).

[21] G. W. Gibbons, M. B. Green, and M. J. Perry, Instantons and seven-branes in type IIB superstring theory, Phys. Lett. B 370, 37 (1996).

[22] H. Liu and A. A. Tseytlin, D3-brane D instanton configuration and $N=4$ superYM theory in constant selfdual background, Nucl. Phys. B553, 231 (1999).

[23] A. Kehagias, On asymptotic freedom and confinement from type IIB supergravity, Phys. Lett. B 456, 22 (1999).

[24] B. Gwak, M. Kim, B. H. Lee, Y. Seo, and S. J. Sin, Holographic D instanton liquid and chiral transition, Phys. Rev. D 86, 026010 (2012).

[25] S. Li and S. Lin, D-instantons in real time dynamics, Phys. Rev. D 98, 066002 (2018).

[26] Z. Q. Zhang, D. F. Hou, and G. Chen, Imaginary potential of moving quarkonia in a D-instanton background, J. Phys. G 44, 115001 (2017).

[27] S. Li, Holographic Schwinger effect in the confining background with D-instanton, arXiv:2005.11955.

[28] A. Karch and E. Katz, Adding flavor to AdS/CFT, J. High Energy Phys. 06 (2002) 043.

[29] A. Baumgartner, Flavor broken $\mathrm{QCD}_{3}$ at large N, J. High Energy Phys. 08 (2020) 145.

[30] G. V. Dunne, Aspects of Chern-Simons theory, arXiv:hepth/9902115.

[31] S. Li and T. Jia, Matrix model and holographic baryons in the D0-D4 background, Phys. Rev. D 92, 046007 (2015).
[32] C. Wu, Z. G Xiao, and D. Zhou, Sakai-Sugimoto model in D0-D4 background, Phys. Rev. D 88, 026016 (2013).

[33] F. Bigazzi, A. L. Cotrone, and R. Sisca, Notes on theta dependence in holographic Yang-Mills, J. High Energy Phys. 08 (2015) 090.

[34] S. Li, A holographic description of theta-dependent YangMills theory at finite temperature, Chin. Phys. C 44, 013103 (2020).

[35] O. Aharony, J. Sonnenschein, and S. Yankielowicz, A holographic model of deconfinement and chiral symmetry restoration, Ann. Phys. (Amsterdam) 322, 1420 (2007).

[36] S. Li and T. Jia, Dynamically flavored description of holographic QCD in the presence of a magnetic field, Phys. Rev. D 96, 066032 (2017).

[37] S. Li, A. Schmitt, and Qun Wang, From holography towards real-world nuclear matter, Phys. Rev. D 92, 026006 (2015).

[38] F. Bigazzi and A. L. Cotrone, Holographic QCD with dynamical flavors, J. High Energy Phys. 01 (2015) 104.

[39] I. R. Klebanov, D. Kutasov, and A. Murugan, Entanglement as a probe of confinement, Nucl. Phys. B796, 274 (2008).

[40] J. Knaute and B. Kämpfer, Holographic entanglement entropy in the QCD phase diagram with a critical point, Phys. Rev. D 96, 106003 (2017).

[41] N. Jokela and J. G. Subils, Is entanglement a probe of confinement?, J. High Energy Phys. 02 (2021) 147.

[42] M. A. Akbari and M. Lezgi, Holographic QCD, entanglement entropy, and critical temperature, Phys. Rev. D 96, 086014 (2017).

[43] S. Ryu and T. Takayanagi, Holographic Derivation of Entanglement Entropy from AdS/CFT, Phys. Rev. Lett. 96, 181602 (2006).

[44] A. W. Peet and J. Polchinski, UV/IR relations in AdS dynamics, Phys. Rev. D 59, 065011 (1999).

[45] R. Emparan, C. V. Johnson, and R. C. Myers, Surface terms as counterterms in the AdS/CFT correspondence, Phys. Rev. D 60, 104001 (1999).

[46] D. Mateos, R. C. Myers, and R. M. Thomson, Thermodynamics of the brane, J. High Energy Phys. 05 (2007) 067.

[47] P. Benincasa, A note on holographic renormalization of probe D-branes, arXiv:0903.4356.

[48] I. Papadimitriou, Holographic renormalization of general dilaton-axion gravity, J. High Energy Phys. 08 (2011) 119.

[49] D. Kharzeev, R. D. Pisarski, and M. H. G. Tytgat, Possibility of Spontaneous Parity Violation in Hot QCD, Phys. Rev. Lett. 81 (1998) 512.

[50] K. Buckley, T. Fugleberg, and A. Zhitnitsky, Can Theta Vacua Be Created in Heavy Ion Collisions?, Phys. Rev. Lett. 84, 4814 (2000).

[51] D. E. Kharzeev, L. D. McLerran, and H. J. Warringa, The effects of topological charge change in heavy ion collisions: 'Event by event $\mathrm{P}$ and $C P$ violation', Nucl. Phys. A803, 227 (2008)

[52] D. Kharzeev, Parity violation in hot QCD: Why it can happen, and how to look for it, Phys. Lett. B 633, 260 (2006).

[53] A. Kitaev and J. Preskill, Topological Entanglement Entropy, Phys. Rev. Lett. 96, 110404 (2006).

[54] M. Levin and X. G. Wen, Detecting Topological Order in a Ground State Wave Function, Phys. Rev. Lett. 96, 110405 (2006). 